\title{
ARTICLE Intestinal epithelial Caspase-8 signaling is essential to prevent necroptosis during Salmonella Typhimurium induced enteritis
}

\author{
Manuela Hefele ${ }^{1}$, Iris Stolzer ${ }^{1}$, Barbara Ruder ${ }^{1}$, Gui-Wei He ${ }^{1}$, Mousumi Mahapatro ${ }^{1}$, Stefan Wirtz ${ }^{1}$, Markus F. Neurath ${ }^{1}$ and \\ Claudia Günther ${ }^{1}$
}

\begin{abstract}
Although induction of host cell death is a pivotal step during bacteria-induced gastroenteritis, the molecular regulation remains to be fully characterized. To expand our knowledge, we investigated the role of the central cell death regulator Caspase- 8 in response to Salmonella Typhimurium. Here, we uncovered that intestinal salmonellosis was associated with strong upregulation of members of the host cell death machinery in intestinal epithelial cells (IECs) as an early event, suggesting that elimination of infected IECs represents a host defense strategy. Indeed, $C a s p 8^{\Delta I E C}$ mice displayed severe tissue damage and high lethality after infection. Additional deletion of Ripk3 or Mlkl rescued epithelial cell death and lethality of Casp $8^{\Delta \mathrm{IEC}}$ mice, demonstrating the crucial role of Caspase-8 as a negative regulator of necroptosis. While Casp $8^{\Delta \mathrm{EEC}} \operatorname{Tnfr}^{-1-}$ mice showed improved survival after infection, tissue destruction was similar to Casp $8^{\Delta \mathrm{EEC}}$ mice, indicating that necroptosis partially depends on TNF-a signaling. Although there was no impairment in antimicrobial peptide secretion during the early phase of infection, functional Caspase- 8 seems to be required to control pathogen colonization. Collectively, these results demonstrate that Caspase- 8 is essential to prevent Salmonella Typhimurium induced enteritis and to ensure host survival by two different mechanisms: maintenance of intestinal barrier function and restriction of pathogen colonization.
\end{abstract}

Mucosal Immunology (2018) 11:1191-1202; https://doi.org/10.1038/s41385-018-0011-x

\section{INTRODUCTION}

The enteroinvasive bacterial pathogen Salmonella enterica serovar Typhi is the most common gastrointestinal pathogen that causes substantial human diseases ranging from gastroenteritis to systemic typhoid fever. ${ }^{1}$ Infection with Salmonella Typhimurium leads to acute, self-limiting intestinal inflammation associated with diarrhea, vomiting, and abdominal pain. ${ }^{2}$ The ability of pathogens, such as Salmonella to colonize and invade the gut is controlled by several factors, including the intestinal microbiota, the gut associated immune system and the intestinal epithelium. ${ }^{3,4}$

In order to gain access into the host system and to initiate systemic infection, Salmonella initially has to cross the intestinal epithelium through enterocytes and $M$ cells located above the Peyer's patches. ${ }^{5-7}$ The infectious cycle of Salmonella Typhimurium includes three important steps: invasion and colonization of the host cell, the establishment of the Salmonella-containing vacuole, a specific niche for replication, ${ }^{8}$ and the escape from the host cell to infect neighboring cells and tissues. ${ }^{9,10}$ All three phases are closely connected to the host cell death machinery, since during the initial step, epithelial cell death needs to be prevented, whereas in the last step Salmonella requires strategies to leave the host cells. ${ }^{11,12}$ Accordingly, previous publications have demonstrated that Salmonella infection in the gut is characterized by increased epithelial cell death., ${ }^{73}$ While epithelial cell death is an important pathological process and the major cause of tissue damage during infection, clearance of infected cells by active cell death has been characterized as a host defense strategy to limit pathogen replication and survival. ${ }^{14}$
Whether Salmonella-induced epithelial cell death represents a host response or rather a bacterial strategy to promote infection still needs to be elucidated. Until recently, apoptosis and pyroptosis mediated by inflammasome activation were considered as principal mechanisms of programmed host cell death in response to infection. ${ }^{15,16}$ Caspase-8 is well known for its function as an initiator caspase during receptor mediated apoptosis. Earlier studies have further implicated non-apoptotic roles for Caspase- 8 in IL-1 $\beta$ maturation and inflammasome regulation in response to infection by microbial pathogens. ${ }^{17-19}$ A recent study further implicated a potential role for Caspase- 8 in the expulsion of intestinal epithelial cells (IECs) by NLRC4 inflammasome activation. ${ }^{20}$ While these studies suggest that Caspase- 8 is a strong mediator of cell death and inflammation, research of the last decade has clearly demonstrated that Caspase- 8 additionally has a rather prosurvival function by suppressing regulated necrosis (necroptosis). The role of regulated necrosis during bacterial infection is currently not well understood. Only a few murine studies reported evidence for necroptosis in infection related disease conditions and these data still remain controversial. While reduced levels of macrophage necroptosis have been demonstrated to increase susceptibility to systemic Salmonella Typhimurium infection in mice lacking interferon alpha receptor $\left(\right.$ Ifnar ${ }^{-1-}$ ), the same study reported that ipk $^{-/-}$ mice showed similar mortality after intravenous infection. ${ }^{21}$ Interestingly, while macrophage necroptosis seems to be only partially dependent on RIPK3, another study observed that RIPK1 kinase-dead mice (K45A mutation) are highly susceptible to

${ }^{1}$ Department of Medicine 1, University of Erlangen-Nuremberg, Erlangen, Germany

Correspondence: Claudia Günther (c.guenther@uk-erlangen.de)

Received: 22 May 2017 Revised: 3 January 2018 Accepted: 14 January 2018

Published online: 8 March 2018 

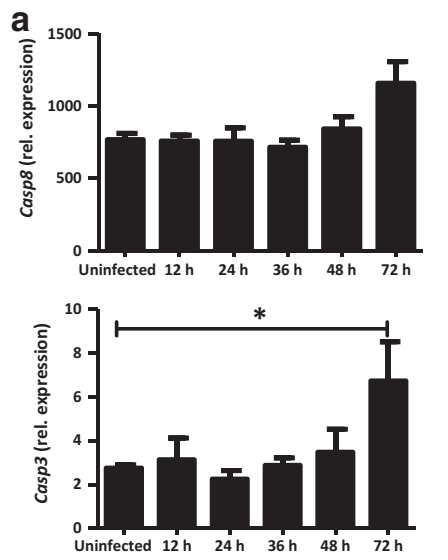

d
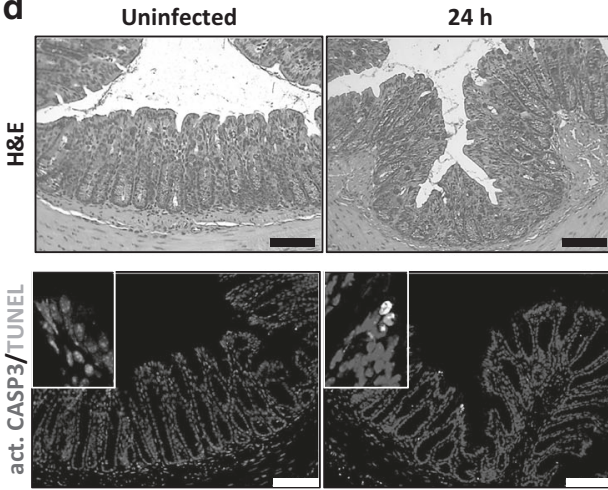

b

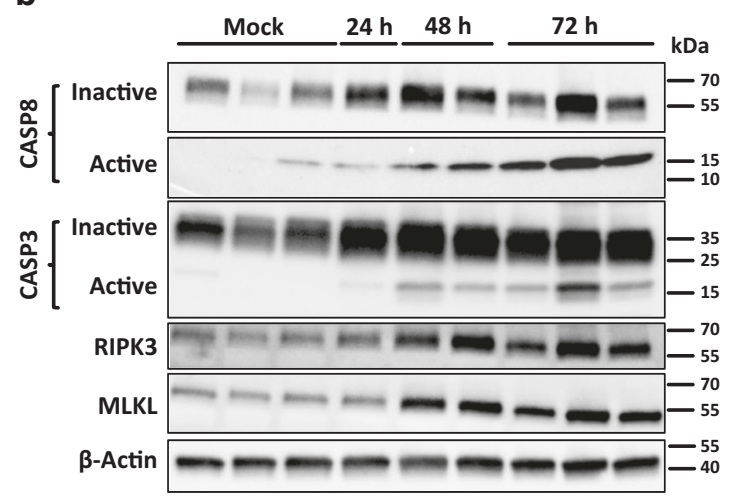

$48 \mathrm{~h}$
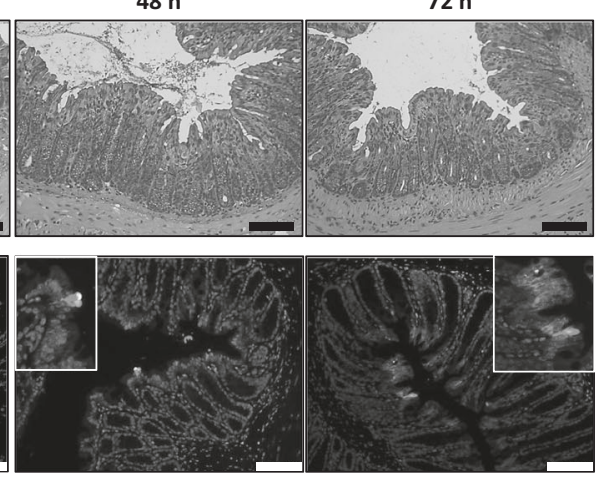
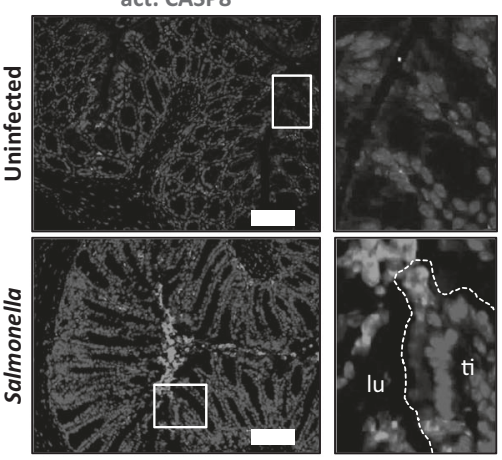

e
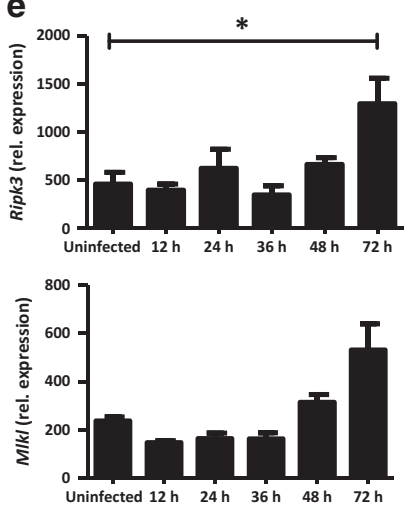

Fig. 1 Infection of wildtype mice with Salmonella Typhimurium provokes caspase activation in the intestinal epithelium. a-e Wildtype (C57BL/ 6) animals were infected orally with $10^{9} \mathrm{CFU}$ of Salmonella Typhimurium ( $\left.\triangle a r o A\right)$ and analyzed at indicated time points ( $n=3$ per time point). Experiments were repeated twice with similar results. a Quantification of colonic Casp8 and Casp3 mRNA expression relative to the expression of Hprt. Data are displayed as mean values $+\mathrm{SD},{ }^{*} p \leq 0.05$. b Western blot analysis of whole tissue lysates (colon) from mock treated and infected wildtype mice using antibodies against full length Caspase-8 (inactive, p55), activated Caspase-8 (p18), full length Caspase-3 (inactive, p35), activated Caspase-3 (p17/19), MLKL (54 kDa) and RIPK3 (57 kDa). $\beta$-Actin was used as a loading control. c Immunohistochemical staining using an antibody against activated CASP8 (red) in colon cross sections 3 days after infection (ti: tissue, lu: lumen; scale bar: 100 um). Hoechst 33342 (blue) was used for counterstaining. d Representative images of colon cross sections stained with H\&E or immunohistochemically with an antibody against activated CASP3 (red) combined with TUNEL assay (green, scale bar: $100 \mu \mathrm{m}$ ). Nuclei were counterstained with Hoechst 33342 (blue). e Gene transcription analysis of colonic Ripk3 and Mlkl. Error bars indicate + SD, gene expression levels are shown relative to Hprt, ${ }^{*} p \leq 0.05$

Salmonella Typhimurium challenge, accompanied by an elevated bacterial burden and reduced macrophage death. ${ }^{22}$ Both studies reported that necroptosis is strongly dependent on interferon (IFN) signaling. Notably, we just recently identified that IFNs induce Mlkl gene transcription via activation of the transcription factor STAT1. ${ }^{23}$ While previous studies indicate that macrophage necroptosis might contribute to systemic Salmonella Typhimurium infection, the role of regulated apoptotic and non-apoptotic epithelial cell death during enteric infection has not been elucidated.

Here, we demonstrate that the early epithelial response to Salmonella Typhimurium infection is characterized by a strong upregulation and activation of key components of the host cell death machinery. By using mice with an IEC specific deletion of the central cell death regulator Caspase-8 (Casp $8^{\Delta / E C}$ mice) we identified that Caspase- 8 is required to ensure a proper host response against Salmonella Typhimurium infection. Mechanistically, we uncovered that Caspase-8 primarily orchestrates the host cell death response by mediating apoptosis and suppression of necroptosis, but did not influence inflammasome activation. Thus, our data show that depending on the cellular context and the physiological situation Caspase- 8 has fundamental functions that are essential to eliminate enteric infection.

\section{RESULTS}

Enteric pathogen infection triggers epithelial cell death

The early epithelial response to bacterial infection includes the expression of pro-inflammatory cytokines, such as tumor necrosis factor (TNF)- $a$ that potentially orchestrate epithelial cell death. ${ }^{24}$ In order to investigate the host cell death mechanism in response to invasive enteric pathogens, we initially evaluated the expression of factors known to be involved in regulated epithelial cell death in mice infected with Salmonella Typhimurium. While the abundance of gene transcripts involved in cell death regulation was not enhanced at early time points during infection, we observed elevated levels of central mediators of apoptosis such as Caspase- 8 and Caspase- 3 , both on mRNA and protein levels $72 \mathrm{~h}$ post-infection (Fig. 1a, b). Similarly, we identified enhanced expression of factors that are well-known for their functions as cell death inducers, such as TNF-a (Figure S1A). Accordingly, Western blot analysis revealed enhanced activation of Caspase- 8 and Caspase-3 already $24 \mathrm{~h}$ post-infection with increasing intensity during the course of infection (Fig. 1b). In line with these data, we observed enhanced epithelial cell shedding accompanied with strong signals for activated caspases in the colon and cecum of infected mice. Notably, caspase activation was most pronounced in cells at the upper part of the crypt during the early phase of infection (Fig. 1c, d). 
a

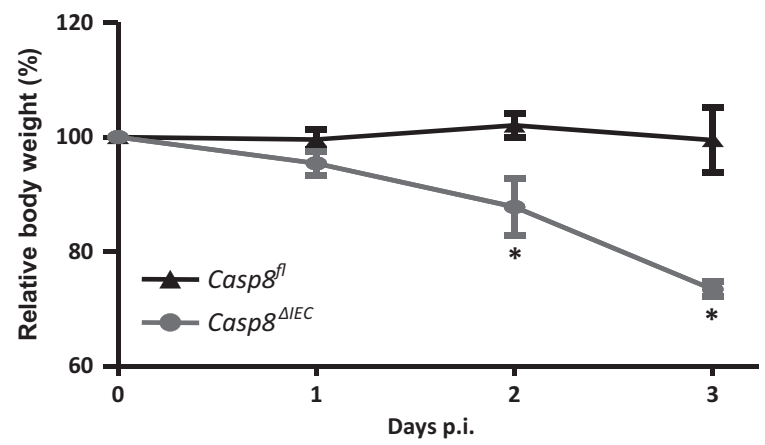

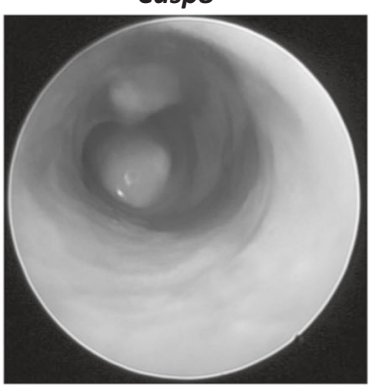

$\operatorname{Casp}^{\Delta I E C}$

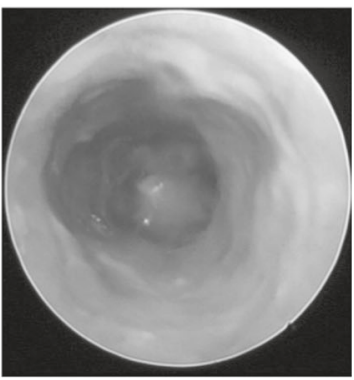

b

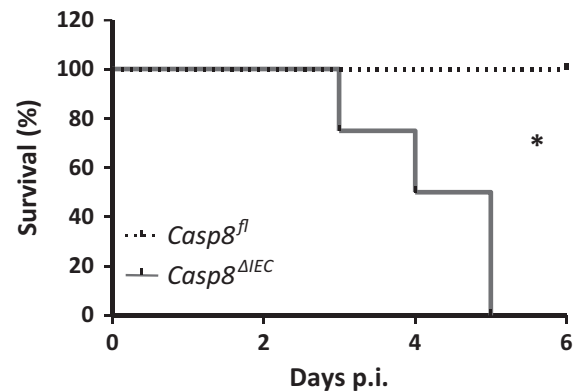

e
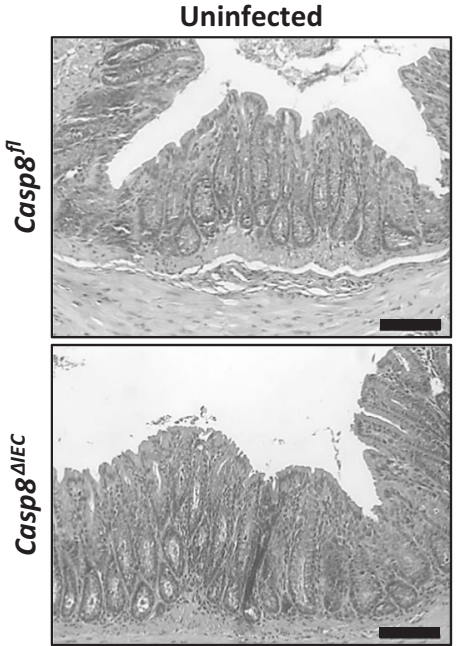

g

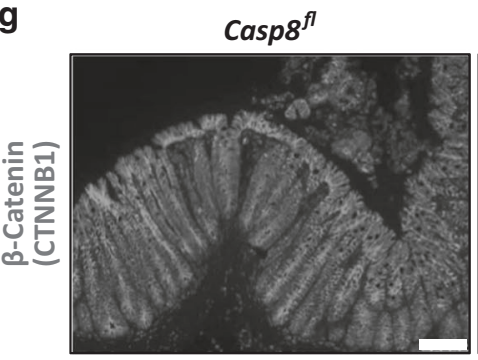

d

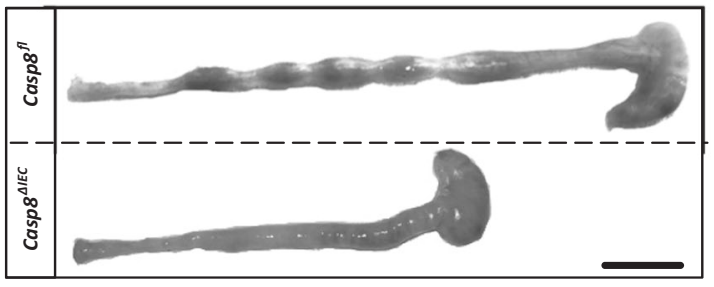

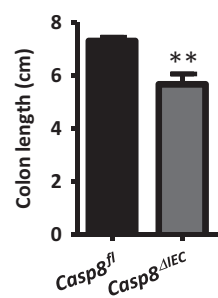

f

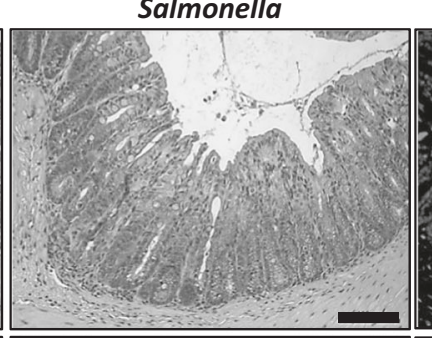

act. CASP3/TUNEL
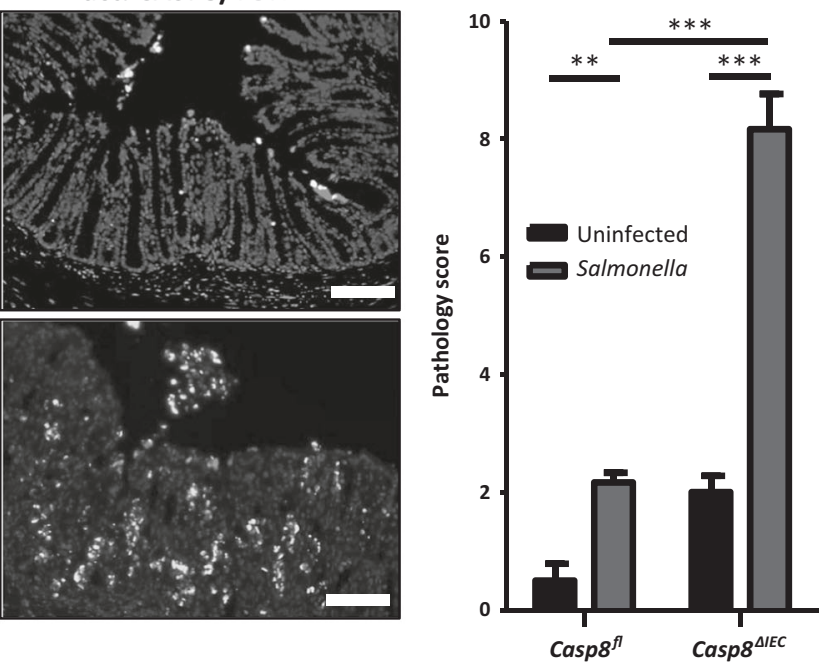

h
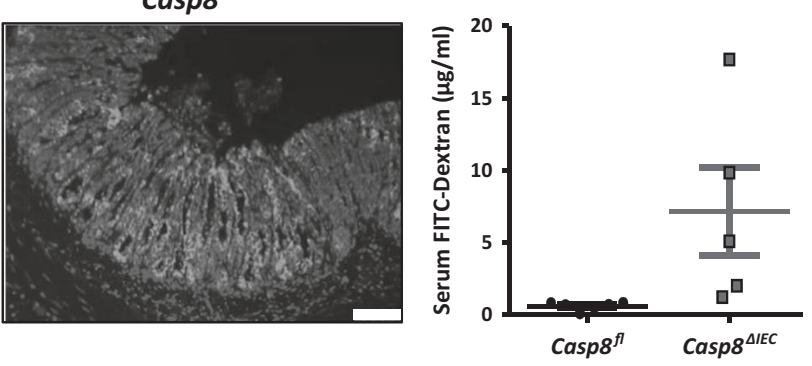

i

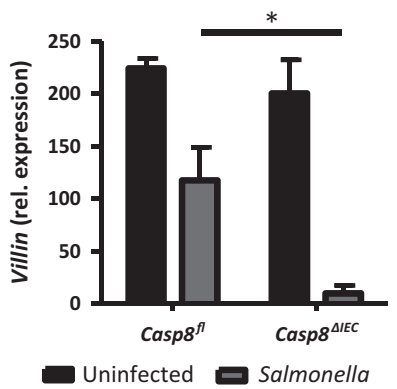

These data suggest that caspase activation is linked to epithelial cell death and cell extrusion in response to Salmonella Typhimurium infection.

Previous studies of our own group and others have demonstrated that Caspase- 8 is a central regulator of both apoptosis and necroptosis. ${ }^{25,26}$ Interestingly, in addition to caspase activation, we observed elevated levels of central necroptosis mediators, including MLKL and RIPK3 during Salmonella Typhimurium infection (Fig. 1b, e). However, we could not observe phosphorylation of RIPK3 or MLKL, suggesting that Caspase-8 signaling is 
Fig. $2 \operatorname{Casp}^{\triangle I E C}$ mice are highly susceptible to Salmonella Typhimurium infection and develop severe enteritis. a-i Control $\left(\right.$ Casp $\left.8^{f l}\right)(n=4)$ and Casp $8^{\triangle I E C}$ mice $(n=4)$ were mock treated or infected with $10^{9}$ CFU of Salmonella Typhimurium ( $\triangle$ aroA, attenuated). $\mathbf{d}-\mathbf{i}$ Data derived at day 3 of infection. Experiments were repeated three times with similar results. a Body weight curve of animals. Data are displayed in percent relative to initial body weight \pm SD. b Kaplan-Meier survival curve of control $\left(\right.$ Casp $\left.8^{f l}\right)$ and Casp $8^{\triangle I E C}$ mice, ${ }^{*} p \leq 0.05$. c Representative endoscopic pictures of control (Casp8 $\left.8^{f}\right)$ and Casp $8^{\triangle I E C}$ mice 4 days after infection. d Representative pictures and statistical analysis of colon shortening ( $n \geq 3$ per group; scale bar: $1 \mathrm{~cm},{ }^{* *} p \leq 0.01$ ). e Representative H\&E stained colon cross sections of mock treated and infected animals (scale bar: $100 \mu \mathrm{m}$ ) and colon cross sections stained with an antibody against activated CASP3 (red) combined with TUNEL assay (green). Nuclei were counterstained with Hoechst 33342 (blue) (scale bar: $100 \mu \mathrm{m}$ ). f Pathology score [maximum: 9] of uninfected and infected colon tissue of control $\left(\right.$ Casp $\left.^{f}\right)$ and Casp $8^{\triangle I E C}$ mice. Data are displayed as mean value $+\mathrm{SD}, n \geq 3$ per group, ${ }^{* *} p \leq 0.01$, ${ }^{* *} p \leq 0.001$. g Representative images of colon cross sections stained with an antibody against $\beta$-Catenin (CTNNB1) (red) and counterstained with Hoechst 33342 (blue) (scale bar: 75 $\mu \mathrm{m})$. $\mathbf{h}$ Serum concentration of orally administered FITC-Dextran in control (Casp $8^{f l}$ ) and Casp $8^{\Delta l E C}$ mice. Serum was separated from the collected blood and the concentration of FITC-dextran was determined by spectrophotofluorometry with an excitation of $485 \mathrm{~nm}$ and an emission of $528 \mathrm{~nm}$ using a serially diluted FITC-dextran standard. Data are displayed as individual values with indicated mean values \pm SD ( $n \geq 5$ per group, data are combined from two independent experiments). i Quantification of colonic Villin mRNA expression ( $n \geq 3$ per group). Error bars indicate $+\mathrm{SD}$, gene expression levels are shown relative to $H p r t,{ }^{*} p \leq 0.05$

essential to prevent necroptosis during Salmonella Typhimurium induced enteritis (data not shown).

Until recently, the analysis of bacterial-epithelial interactions in the intestine has been hampered by the lack of reliable intestinal epithelium culture systems. In order to study the direct host-microbial interaction between IECs and Salmonella Typhimurium, we infected primary intestinal organoids in vitro. To visualize infection of organoids with Salmonella Typhimurium we used a green fluorescent protein (GFP) expressing strain of Salmonella Typhimurium (Figure S1B). Four hours post-infection we observed elevated mRNA expression of genes known to be involved in cell death regulation. Similar to the data obtained in vivo, members of both apoptosis and necroptosis were profoundly upregulated in infected organoids, indicating that both signaling pathways might influence epithelial cell death and barrier function in response to enteric infection (Figure S1C). Induction of epithelial cell death has been characterized as a host defensive mechanism to limit infection by enteric pathogens. Thus, increment of epithelial turnover is required to facilitate the repair of epithelial injuries and to decrease intestinal permeability induced by pathogens. Accordingly, we observed upregulation of the proliferation marker Ki67 in infected organoids (Figure S1D). The innate immune system provides defense against invasive pathogens by inducing a variety of inflammatory responses, including expression of inflammatory cytokines and antimicrobial peptide (AMP) secretion. ${ }^{27}$ To determine the immunological relevance of IECs we next evaluated the expression of intestinal epithelial derived cytokines in response to Salmonella Typhimurium infection. Infection of intestinal organoids resulted in a strong induction of pro-inflammatory cytokines, including Ifnb, I/1a/b, and Tnfa (Figure S1E).

In summary these data implicate a potential role of epithelial Caspase- 8 at the crossroad between IEC survival and regulated cell death including apoptosis and necroptosis in response to Salmonella Typhimurium.

Caspase- 8 orchestrates epithelial apoptosis and prevents barrier dysfunction in response to Salmonella infection

In order to study a potential contribution of Caspase-8 to the pathogenesis of gastrointestinal infections, we subjected Cas$p 8^{\Delta I E C}$ mice and control littermates (Casp $8^{f l}$ mice) to the streptomycin mouse model for Salmonella Typhimurium enteritis. Casp $8^{\triangle I E C}$ mice are maintained on a C57BL/6 background and previous studies have demonstrated that $\mathrm{C} 57 \mathrm{BL} / 6$ are highly susceptible to infection with wild-type Salmonella Typhimurium due to an amino acid substitution in the natural resistanceassociated macrophage protein-1 (nramp ${ }^{D 169}$ ) resulting in a nonfunctional protein. ${ }^{28}$ Since we have previously shown that Casp $8^{\triangle I E C}$ mice are highly susceptible to lipopolysaccharide (LPS) administration and dextran sulfate sodium-induced colitis, we infected Casp $8^{\triangle I E C}$ mice and control littermates with the attenuated Salmonella Typhimurium $\triangle$ aroA mutant. ${ }^{25,26}$ Although the $\triangle a r o A$ mutant strain has impaired replication rates and thus reduced virulence, this strain sufficiently colonizes the colon and cecum and triggers pronounced inflammation and fibrosis in infected mice. ${ }^{28,29}$ While oral administration of the attenuated Salmonella strain had only a mild effect on wildtype mice, Casp $8^{\triangle I E C}$ mice displayed severe weight loss and high mortality already in the initial phases of infection (Fig. 2a, b). Highresolution endoscopy revealed severe signs of inflammation in the colon of Casp8 ${ }^{\triangle l E C}$ mice, including thickening of the bowel wall, reduced translucency, loss of regular vessel structure and fibrin extravasation (Fig. 2c). Ex vivo assessment of the infected colons further demonstrated a significantly reduced colon length compared to littermate controls (Fig. 2d). Accordingly, histological analysis revealed severe tissue destruction, accompanied by pronounced edema of the submucosa, massive epithelial cell death and inflammatory infiltrates in both the cecum and colon of $C a s p 8^{\triangle I E C}$ mice (Figs. 2e, f, 3a, b, S3A, B). While cell death in control mice was restricted to the upper part of the crypt and gut lumen, $\operatorname{Casp} 8^{\triangle I E C}$ mice showed massive cell death along the entire crypt axis both in the colon and cecum (Figs. 2e, 3c). $\beta$-Catenin immunostaining in these samples identified that massive cell death in Casp $8^{\triangle I E C}$ mice finally culminated in a general destruction of the intestinal epithelium (Fig. 2g, 3c). To investigate whether deficiency of Caspase-8 increases intestinal permeability, we next subjected Salmonella infected mice to fluorescein isothiocyanate-dextran (FITC-dextran) oral gavage. We noted highly elevated FITC serum levels in Casp $8^{\triangle I E C}$ mice as compared to control mice, suggesting a breakdown of intestinal barrier function (Fig. 2h). Loss of epithelial barrier was further confirmed by significantly reduced mRNA levels of the epithelial cell marker Villin (Fig. 2i). Notably, while we could not observe tissue destruction or inflammation in the small intestine of control mice, Casp $8^{\triangle I E C}$ mice also developed severe enteritis associated with massive epithelial erosions in the ileum (Fig. 3d).

To better characterize the kinetics behind Salmonella induced tissue destruction in $\operatorname{Casp} 8^{\triangle I E C}$ mice, we investigated the gut morphology at different time points following infection $(24,48$, and $72 \mathrm{~h}$ ). While $24 \mathrm{~h}$ post infection Casp $8^{\Delta / E C}$ mice showed highly increased expression of the pro-inflammatory marker S100a9 (S2E), both control and Casp8 $8^{\triangle I E C}$ mice showed no remarkable changes in tissue morphology (Figure S2A, B). Epithelial cell death as visualized by TUNEL staining and barrier break down, demonstrated by $\beta$-Catenin staining and quantification of Villin mRNA levels, were detectible in Casp $8^{\Delta / E C}$ mice, but not in control littermates, starting from $48 \mathrm{~h}$ post infection in the colon and cecum (Figures S2C, D, S3C, D).

In summary these data demonstrate that Caspase- 8 is essential to strengthen barrier function in response to Salmonella Typhimurium induced enteritis and that Casp $8^{\triangle I E C}$ mice provide 


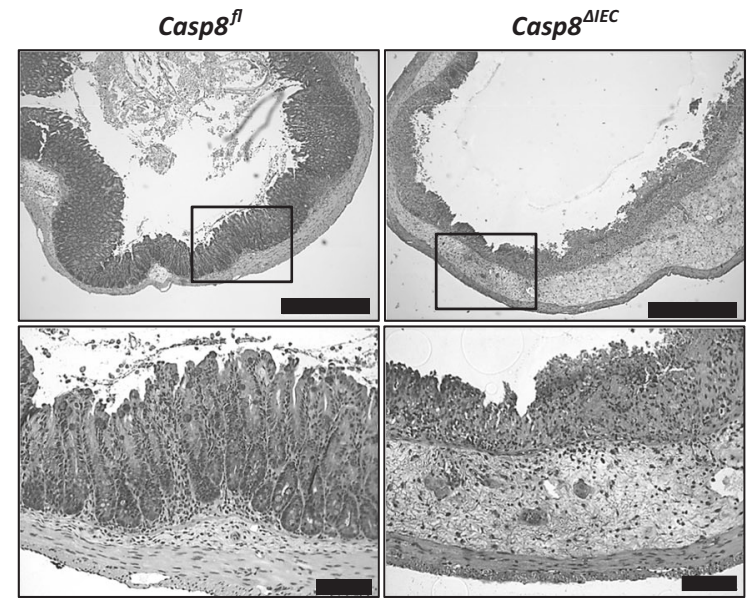

C
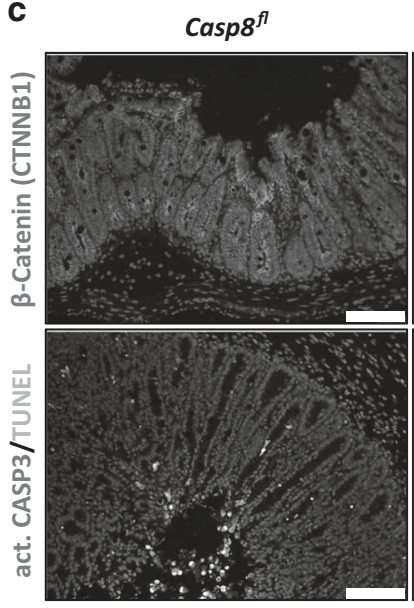
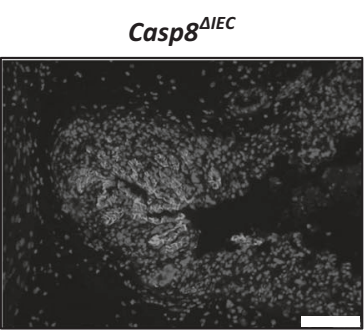

b

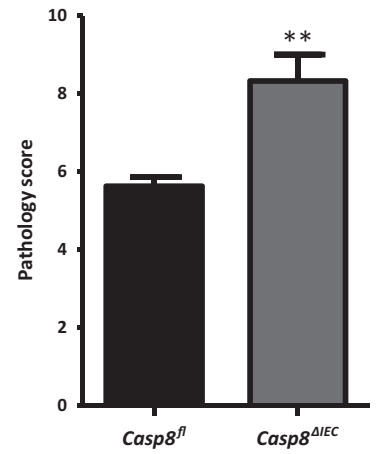

d Terminal ileum

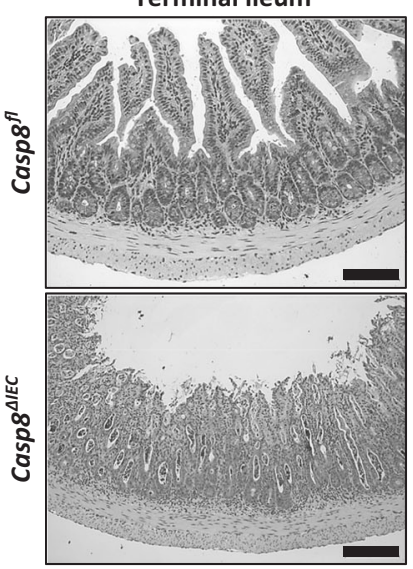

Fig. 3 Infection with Salmonella Typhimurium causes severe cecitis in Casp $8^{\triangle I E C}$ mice. a-d Control $\left(\operatorname{Casp} 8^{f f}\right)(n=4)$ and Casp $8^{\triangle I E C}$ mice $(n=3)$ were mock treated or infected with $10^{9} \mathrm{CFU}$ of Salmonella Typhimurium ( $\triangle$ aroA, attenuated). Mice were sacrificed after 3 days. Tissue samples from cecum and terminal ileum were harvested and stored for further analysis. Experiments were repeated three times with similar results. a Representative images of H\&E stained infected cecum cross sections (scale bar: $500 \mu \mathrm{m}$, inset scale bar: $100 \mu \mathrm{m})$. b Pathology score [maximum: 9] of infected ceca represented as mean values $+S D,{ }^{* *} p \leq 0.01$. c Representative images of cecum cross sections stained with antibodies against $\beta$-Catenin (CTNNB1) (red) or activated CASP3 (red) in combination with TUNEL (green). Hoechst 33342 (blue) was used for counterstaining of nuclei (scale bar: $100 \mu \mathrm{m}$ ). d Representative images of H\&E stained cross section of the infected terminal ileum (scale bar: $100 \mu \mathrm{m})$

a powerful tool to elucidate disease mechanism underlying inflammation during enteric infection.

Epithelial Caspase-8 expression is essential to restrict colonization during Salmonella Typhimurium infection

IECs constitute important host defense mechanisms to control access and survival of both commensal bacteria and pathogens. In order to study if intestinal epithelial Caspase-8 is essential to control colonization of the intestine, we infected mice with a wildtype Salmonella strain to exclude strain-dependent differences in replication efficiency. To better visualize bacterial loads, we used a bioluminescent wildtype Salmonella strain (UK1). ${ }^{28}$ We observed inflammatory lesions and increased epithelial extrusion in the colon of wildtype animals (Figure S4A). Of note Casp $8^{\Delta I E C}$ mice showed massive tissue destruction and significantly decreased survival compared to control littermates, similar to the data obtained using the attenuated $\triangle a r o A$ mutant strain (Figure S4). We observed an enhanced and equal colonization of the intestine at day 2 post infection in control and Casp $8^{\triangle I E C}$ mice (Fig. 4a). However, while control littermates successfully defended infection as demonstrated by ex vivo imaging of the gastrointestinal tract at day $4, \operatorname{Casp} 8^{\Delta \mathrm{IEC}}$ mice contained a high burden of Salmonella in the cecum and colon (Fig. 4b). These data implicate that epithelial
Caspase-8 signaling is an essential host defense mechanism to control intestinal colonization during Salmonella infection.

Previous studies have demonstrated that low expression of mucin glycoproteins was associated with a reduced thickness of the mucus layer which enables Salmonella to directly attach to the intestinal epithelium, to invade and finally to build microcolonies. $^{30,31}$ Whereas Salmonella infection induced enhanced amounts of Mucin2 (MUC2) glycoproteins in wildtype animals, Casp $8^{\triangle I E C}$ mice failed to increase Muc2 mRNA transcription and even showed a dramatic depletion of the mucus layer at later time points of infection (Fig. 4c, S5A). Hence, we hypothesized that depletion of the mucus layer facilitates commensal and noncommensal bacteria to directly attach to the intestinal epithelium and to translocate from the luminal side into subepithelial areas of $C a s p 8^{\triangle I E C}$ mice. Accordingly, fluorescence in situ hybridization with a eubacterial rRNA-specific probe revealed strong bacterial signals within the mucosa of Casp $8^{\triangle I E C}$ mice (Fig. 4d). In sharp contrast to $\operatorname{Casp} 8^{\triangle I E C}$ mice, bacteria remained most pronounced in the lumen of control mice (Fig. 4d). This was accompanied by massive infiltration of immune cells and an elevated expression of pro-inflammatory cytokines, like $116,5100 a 9$, and interferons, in Casp $8^{\triangle I E C}$ mice (Fig. 4e, S5B, C). We further observed a dramatic systemic spread of mucosal bacteria into other visceral organs 

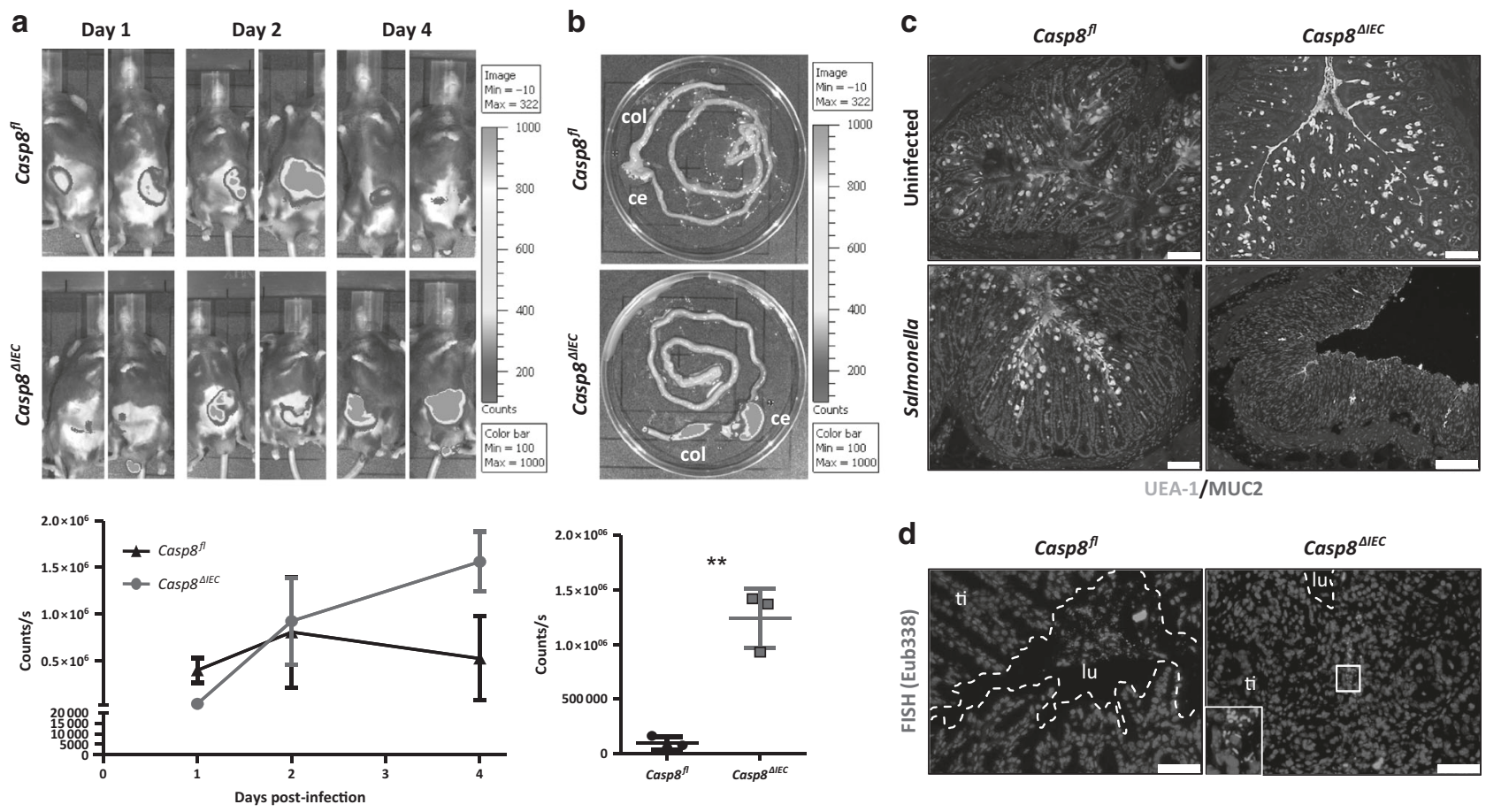

d
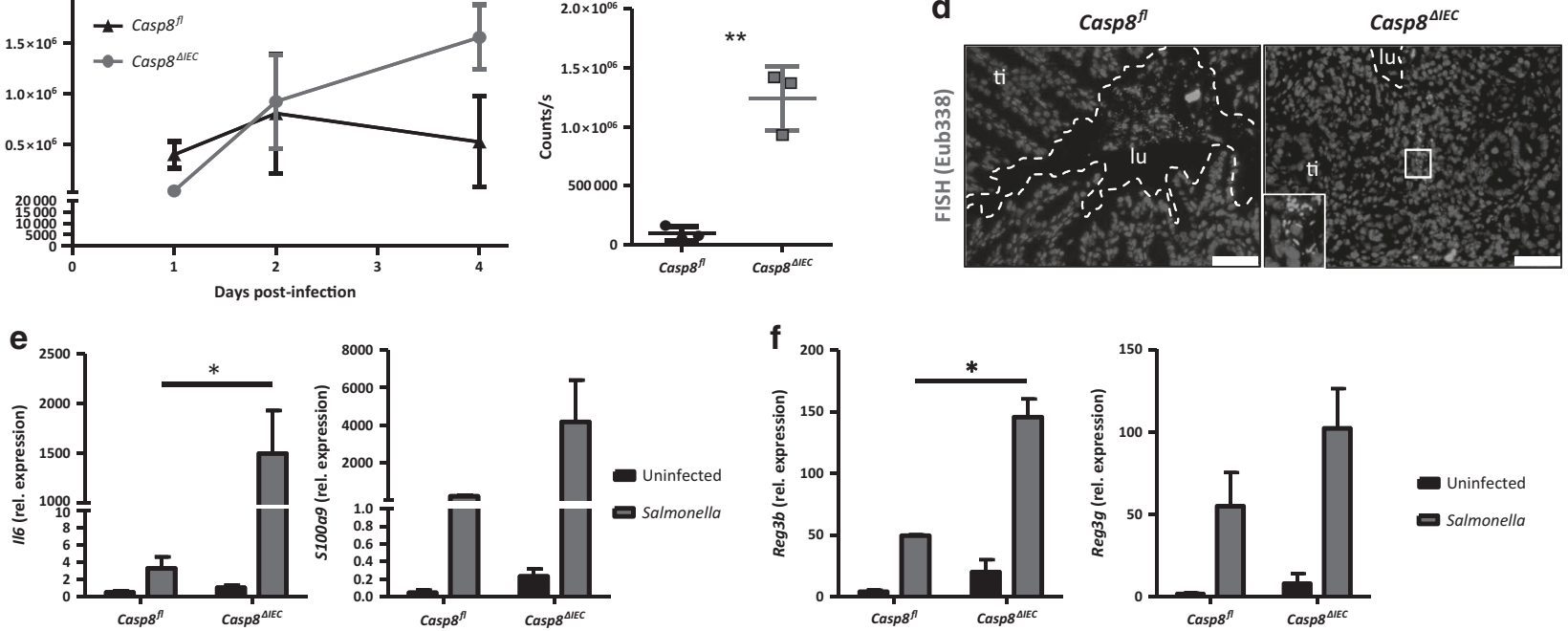

Fig. 4 Lack of epithelial Caspase-8 facilitates barrier break down and systemic spread of mucosal microbes. $\mathbf{a}$, b Control $\left(\right.$ Casp $\left.8^{f l}\right)(n=3)$ and Casp $8^{\triangle I E C}(n=3)$ mice were infected with $10^{9} \mathrm{CFU}$ of a bioluminescent strain of Salmonella Typhimurium (UK-1). Red areas represent regions with highest bacterial burden. Experiments were repeated three times with similar results. a Representative visualization and quantification of Salmonella Typhimurium burden in control and Casp $8^{\Delta I E C}$ mice at indicated time points. Data are displayed as mean values \pm SD. b Representative ex vivo imaging and quantification of bioluminescent Salmonella Typhimurium in the gut of infected mice (ce cecum, col colon). Data are displayed as individual values with indicated mean values \pm SD, ${ }^{* *} p \leq 0.01$. c-f Control $\left(\right.$ Casp $\left.8^{f f}\right)$ and Casp $8^{\Delta I E C}$ mice $(n \geq 3$ per group) were infected with Salmonella Typhimurium ( $\triangle a r o A$, attenuated) and analyzed at day 2 (f) or day 3 (c-e) of infection. Experiments were repeated three times with similar results. c Representative images of colon cross sections from unchallenged and infected animals stained with an antibody against MUC2 (red) combined with UEA-1 (green, scale bar: $75 \mu \mathrm{m}$ ). Hoechst 33342 (blue) was used for counterstaining of nuclei. d Representative images of fluorescence in situ hybridization (FISH) with a universal eubacterial probe (red) in colon cross sections of infected mice (ti: tissue, lu: lumen; scale bar: $50 \mu \mathrm{m}$ ). Hoechst 33342 (blue) was used for counterstaining. e Quantification of colonic Il6 and S100a9 gene expression relative to the expression of Hprt. Data are represented as mean values $+\mathrm{SD}, n \geq 3,{ }^{*} p \leq 0.05$. $\mathbf{f}$ Quantification of colonic Reg $3 b$ and Reg $3 g$ mRNA relative to the expression of Hprt, $n \geq 3,{ }^{*} p \leq 0.05$

such as mesenteric lymph nodes, liver, and spleen in Casp $8^{\triangle I E C}$ mice, suggesting that barrier defects and translocation of bacteria are responsible for the lethality of these mice (Figure S5D).

Invasion of IECs by Salmonella triggers an inflammatory response, leading to the release of AMPs. Previously, we have shown that Casp $8^{\Delta I E C}$ mice display defects in the antimicrobial defense due to a reduced expression of AMPs. ${ }^{26}$ Therefore, we next evaluated if enhanced bacterial colonization of Salmonella Typhimurium in Casp8 $8^{\triangle I E C}$ mice is linked to defects in enteric AMP production. Analysis of AMP expression revealed a strong increase in Reg $3 b$ and Reg $3 g$ transcripts in response to Salmonella infection in both control and Casp8 ${ }^{\triangle l E C}$ mice 2 days post infection (Fig. $4 \mathrm{f}$ ). Moreover, Casp $8^{\triangle I E C}$ mice displayed higher Reg gene expression, possibly as a host response adapted to the elevated bacterial burden. In order to investigate the direct interaction and host response of IECs to enteric pathogens, we infected intestinal organoids derived from Caspase- 8 proficient and deficient animals with Salmonella Typhimurium in vitro. Similar to the results obtained in vivo, both organoid cultures responded with strong induction of Reg3g mRNA expression, suggesting that Caspase-8 deficiency does not impair expression of AMPs (Figure S5E). These data indicate that at later time points during the infection, deficiency in AMP secretion caused by loss of IECs along with depletion of the mucus layer, allows Salmonella to colonize more efficiently in Casp $8^{\triangle I E C}$ mice.

In summary these data demonstrate that Caspase- 8 is essential to promote host resistance to Salmonella Typhimurium by orchestrating the gut response to control bacterial burden and to prevent tissue colonization.

Distinct factors trigger necroptosis orchestrated by MLKL and RIPK3 during Salmonella infection

Apart from its function as a central regulator of apoptosis, recent studies identified novel roles of Caspase-8 downstream of the NLRC4 inflammasome for modulating IL-1 $\beta$ production and intestinal epithelial cell extrusion. ${ }^{20,32}$ Notably, infected Caspase- 


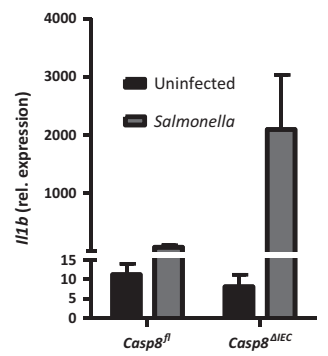

b
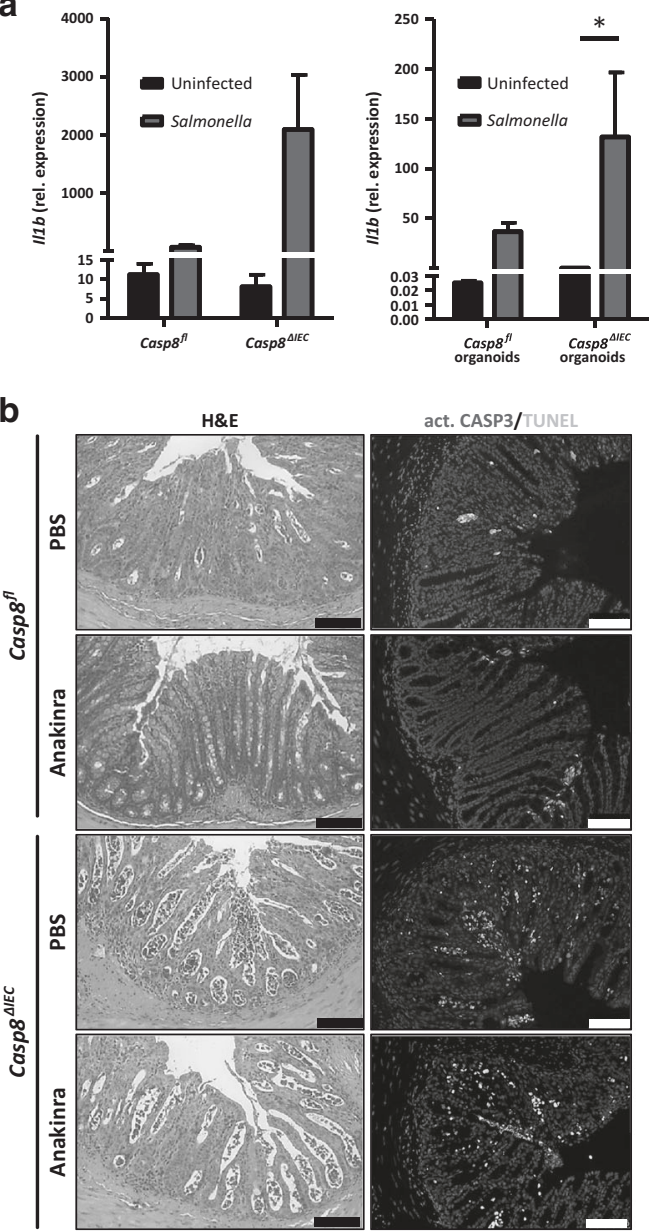

C

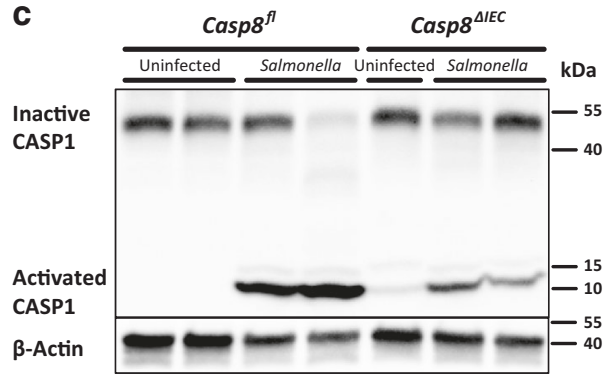

d

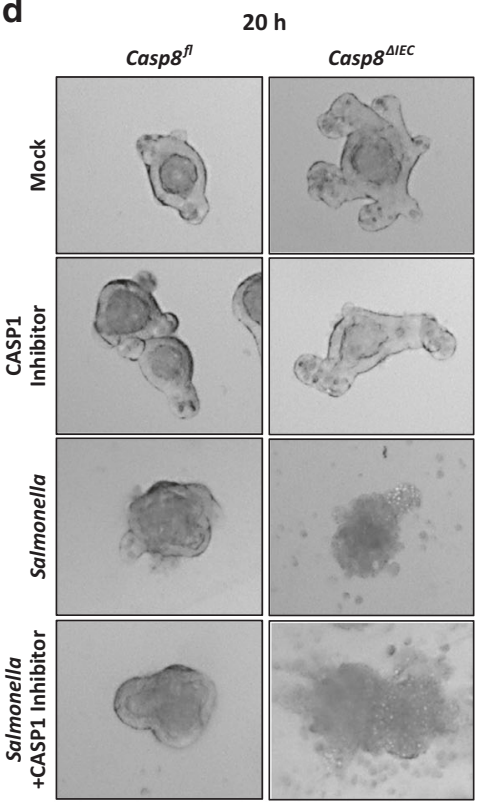

Fig. 5 Blocking of IL-1 signaling does not improve barrier function or survival of Casp $8^{\triangle I E C}$ mice. a-c Control (Casp $\left.8^{f f}\right)$ and Casp $8^{\triangle I E C}$ mice were mock treated or infected with $10^{9} \mathrm{CFU}$ of Salmonella Typhimurium ( $\triangle$ aro $A$, attenuated). Mice were sacrificed at day 3 post-infection. a Quantification of $I 11 b$ mRNA in colonic tissue ( $n \geq 3$ per group) and in in vitro organoid cultures $\left(n=3\right.$ per group) relative to Hprt, + SD, ${ }^{*} p \leq$ 0.05. b Control $\left(\operatorname{Casp}^{f f}\right)$ and Casp $8^{\triangle I E C}$ mice were treated intraperitoneally with a daily dose of PBS or Anakinra during infection with Salmonella Typhimurium ( $\triangle a r o A$, attenuated) and analyzed at day 3 ( $n=3$ per group). Experiments were repeated two times with similar results. Representative images of H\&E stained colon cross sections and immunohistochemical staining with an antibody against activated CASP3 (red) and TUNEL (green). Hoechst 33342 (blue) was used for counterstaining of nuclei (scale bar: $100 \mu \mathrm{m}$ ). c Western blot analysis of colonic intestinal epithelial scrapings incubated with an antibody targeting the pro-form and the activated p10 fragment of Caspase-1. $\beta$-Actin was used as a loading control. d Representative images of mock treated and infected intestinal organoids derived from control (Casp $\left.8^{f}\right)$ and Casp $8^{\triangle I E C}$ mice $20 \mathrm{~h}$ after infection. For inhibition of Caspase-1, organoids were treated with $50 \mu \mathrm{M}$ Belnacasan (VX-765). Propidium lodide (red) visualizes dead epithelial cells

8 deficient IECs exhibited a hyperactive production of $/ 11 b$ mRNA in vivo and in vitro compared to control cells (Fig. 5a). Although $\mathrm{IL}-1 \beta$ is not directly required for cell death, IL-1 $\beta$ production contributes to the inflammatory response elicited by cells undergoing Caspase-1 mediated pyroptosis. In order to investigate whether increased IL-1 $\beta$ production is causally linked to excessive tissue destruction and inflammation in Casp $8^{\triangle I E C}$ mice during Salmonella Typhimurium infection, we treated $\operatorname{Casp}^{\triangle I E C}$ mice and control animals with a daily dose of the recombinant IL-1 receptor antagonist Anakinra, starting one day before the infection. While administration of Anakinra ameliorated pathogen induced inflammation and body weight loss in control animals, tissue destruction and barrier break down in $\operatorname{Casp} 8^{\triangle I E C}$ mice was not dependent on IL-1 signaling (Fig. 5b, S6A). These data demonstrate that IL-1 receptor signaling does not substantially contribute to barrier dysfunction in Casp $8^{\triangle I E C}$ mice. As mentioned above, Caspase- 8 has also been shown to act downstream of the NLRC4 inflammasome. Therefore, we next investigated IEC inflammasome activation following Salmonella infection. In line with previous publications, we observed Caspase-1 processing in response to Salmonella infection in epithelial scrapings of control and Casp $8^{\triangle I E C}$ mice (Fig. 5c). Notably, Salmonella infection potently induced Caspase-1 cleavage to a similar extend in Casp $8^{\Delta I E C}$ mice and control littermates (Fig. 5b), suggesting that Caspase-1 mediated pyroptosis cannot explain excessive epithelial cell death as observed in $C a s p 8^{\triangle I E C}$ mice. To provide functional evidence, we infected organoids derived from control and Casp $8^{\Delta l E C}$ mice in vitro. Notably, we observed a more robust Salmonella-induced cytotoxicity accompanied with IEC death in Caspase-8 deficient organoids. Importantly, our data suggest that Caspase- 1 is not involved in death of Caspase-8 deficient intestinal epithelial cells, since co-incubation of Caspase- 8 deficient organoids with the potent and selective Caspase-1 inhibitor belnacasan (VX-765) could not restore viability of epithelial cells in response to Salmonella infection (Fig. 5d, S6B). These data suggest that Caspase-1 mediated pyroptosis does not substantially contribute to tissue injury observed in $\operatorname{Casp} 8^{\triangle I E C}$ mice. In order to address the contribution of Caspase-8 to intracellular replication of Salmonella Typhimurium, we infected Caspase- 8 proficient and deficient HT29 human colorectal cancer cells with Salmonella and evaluated 

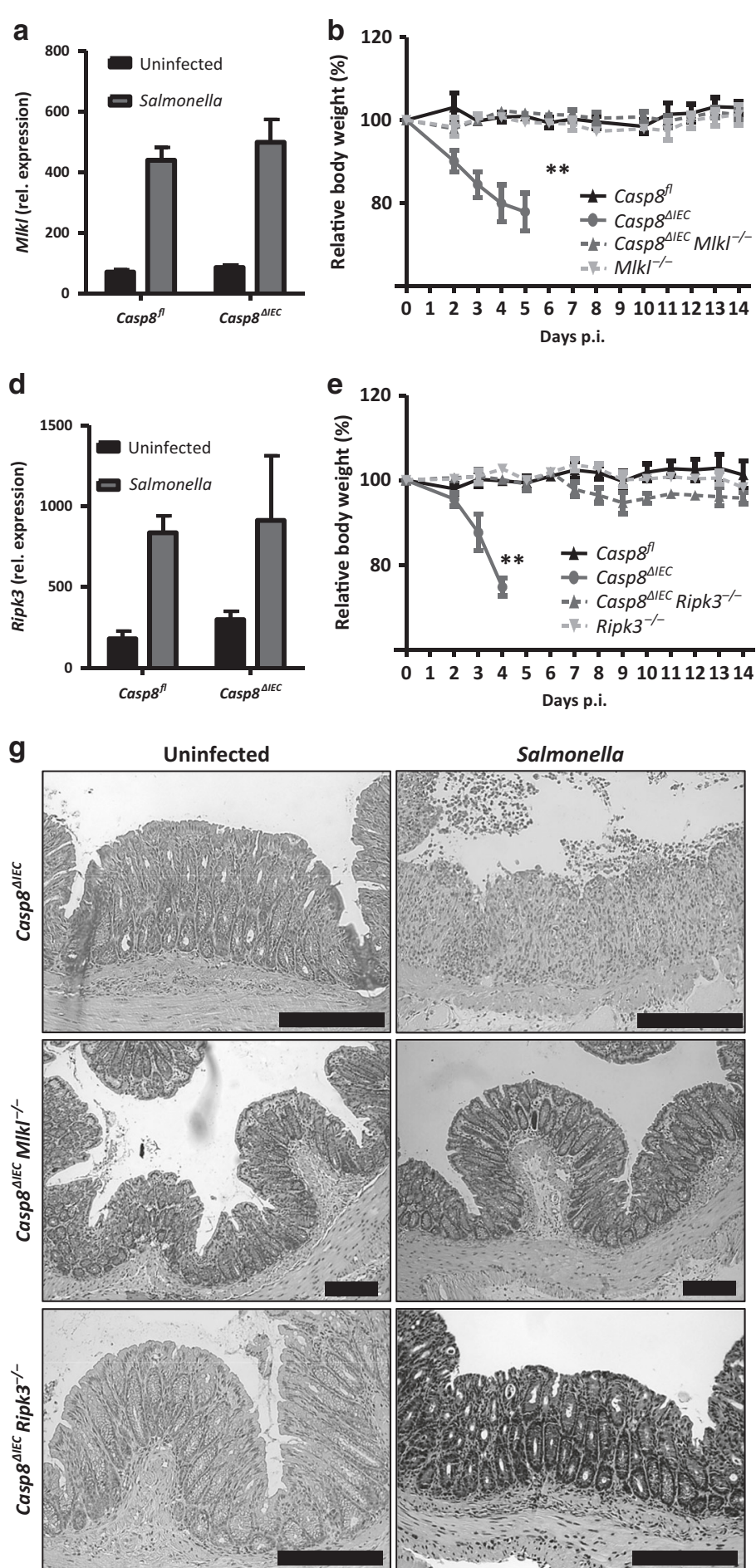

Fig. 6 Genetic ablation of necroptosis mediators restores barrier function and ensures survival of Casp $8^{\triangle I E C}$ mice. a-h Mice were mock treated or infected with $10^{9} \mathrm{CFU}$ of Salmonella Typhimurium ( $\triangle a r o A$, attenuated). $\mathbf{a}, \mathbf{d}, \mathbf{g}, \mathbf{h}$ Mice were analysed at day 3 of infection. Experiments were repeated two times with similar results. a Colonic expression of MlkI mRNA in mock treated and infected control (Casp $8^{f l}$ ) and Casp $8^{\Delta I E C}$ mice.

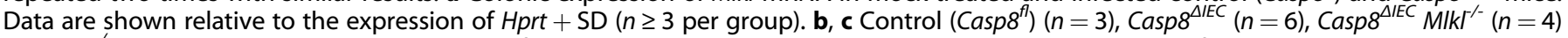
and $\mathrm{Mlkl}^{\prime-}(n=3)$ mice were mock treated or infected with Salmonella Typhimurium $(\triangle a r o A)$. b Body weight of the animals is displayed relative to initial body weight $\pm S D,{ }^{* *} p \leq 0.01$. c Kaplan-Meier curve for survival of the animals after infection, ${ }^{*} p \leq 0.05$. d Colonic expression of Ripk3 mRNA in mock treated and infected control $\left(\operatorname{Casp} 8^{f}\right)$ and Casp $8^{\triangle I E C}$ mice. Data are shown relative to the expression of $H p r t+\operatorname{SD}(n \geq 3$ per

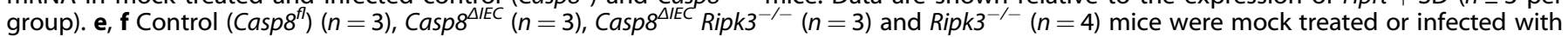
Salmonella Typhimurium $(\triangle a r o A)$. e Body weight of the animals is displayed relative to initial body weight $\pm S D$, ** $p \leq 0.01$. $f$ Kaplan-Meier curve for survival of the animals after infection, ${ }^{*} p \leq 0.05$. g Representative H\&E stained colon cross sections of mock treated or infected Casp $8^{\Delta I E C}$, Casp $8^{\triangle I E C}$ MIkI $^{-1-}$, Casp $8^{\triangle I E C}$ Ripk $3^{-1-}$ animals (scale bar: $200 \mu \mathrm{m}$ ). h Pathology score of infected colon tissue collected from mock

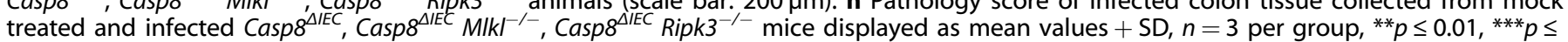
0.001 

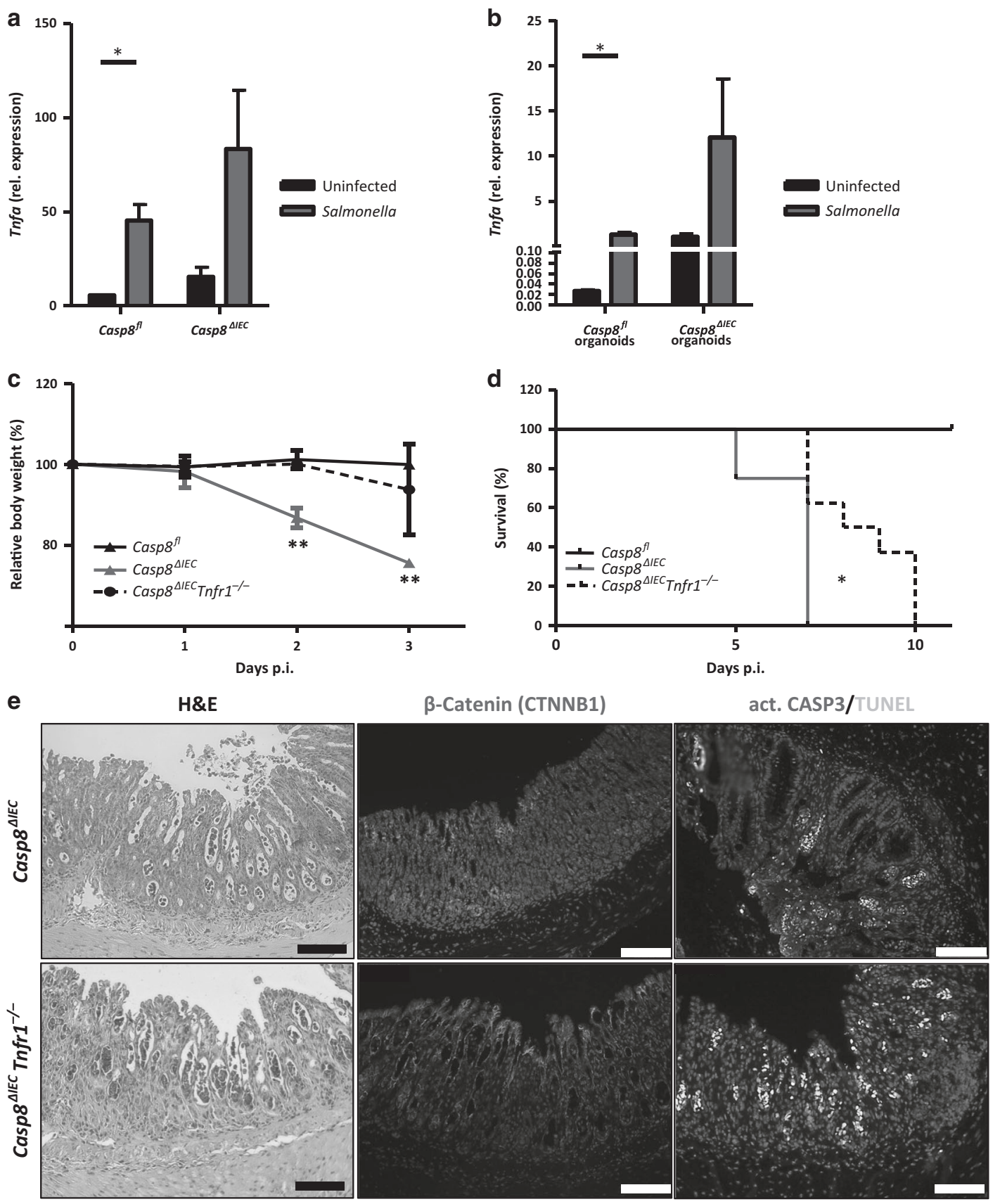

Fig. 7 Lethality of Casp $8^{\triangle I E C}$ mice partially depends on TNF- $\alpha . \mathbf{a}, \mathbf{b}$ Quantification of Tnfa mRNA in colonic tissue of mice infected for 4 days $(n \geq 3)(a)$ and in organoids infected for $4 \mathrm{~h}(n \geq 3)$ (b) with Salmonella Typhimurium ( $\triangle$ aroA, attenuated). Error bars indicate + SD, gene expression levels are shown relative to Hprt, ${ }^{*} p \leq 0.05$. Experiments were repeated three times with similar results. c-e Control $\left(C a s p 8^{f f}\right)(n=4)$, Casp $8^{\triangle I E C}(n=4)$, and Casp $8^{\triangle I E C} \operatorname{Tnfr}^{-\prime-}(n=8)$ mice were mock treated or infected with $10^{9}$ CFU of Salmonella Typhimurium $(\triangle a$ aroA, attenuated). Experiments were repeated two times with similar results. c Body weight of the animals is displayed relative to initial body weight $\pm S D,{ }^{* *} p \leq 0.01$. d Kaplan-Meier curve for survival of the animals after infection, $n \geq 4$ per group, ${ }^{*} p \leq 0.05$. e Representative images of colon cross sections of infected mice stained with H\&E, $\beta$-Catenin (CTNNB1) (red) or activated CASP3 (red) in combination with TUNEL (green). Hoechst 33342 (blue) was used for counterstaining of nuclei (scale bar: $100 \mu \mathrm{m}$ )

the intracellular bacterial burden. Interestingly, while we could not observe differences in the viability of both cell lines, Caspase-8 deficient HT-29 cells showed a significantly higher burden of intracellular bacteria compared to wildtype cells (Figure S6C). Similar results could be obtained in murine colon adenocarcinoma cells (MC38 cells), which were treated with the Caspase-8 inhibitor Z-IETD-FMK (Figure S6D). To ascertain the influence of Caspase-1 to intracellular replication in Caspase-8 deficient epithelial cells, we infected HT-29 (Caspase-8 proficient and deficient) cells in the presence or absence of a Caspase- 1 inhibitor. We could not observe significant differences (Figure S6E). In summary these data suggest that during intestinal infection, Caspase- 8 in IECs is essential to protect from pathogen invasion independent of Caspase-1.

Our previous publications have identified that Caspase- 8 has an important function as a negative regulator of necroptosis in response to pro-inflammatory cytokines such as TNF-a, but also microbial molecules (LPS), suggesting that IECs in 
$\operatorname{Casp} 8^{\triangle I E C}$ mice undergo necroptosis in response to bacterial infections. $^{25,33}$

While IEC death in infected control mice was associated with strong activation of Caspase-3, we observed massive TUNEL positive IECs that stained negative for activated caspases in Casp $8^{\triangle I E C}$ mice, suggesting that barrier dysfunction in these mice might be due to necroptosis of IECs (Fig. 2e, 3c, S3D). Accordingly, intestinal expression of central regulators of necroptosis, such as Mlkl and Ripk3 was highly increased in challenged mice (Fig. 6a, d). Of note infection of organoids with Salmonella Typhimurium revealed that $M l k l$ and Ripk3 mRNA was in particular upregulated in intestinal epithelial cells (Figure S7A). In line with these results, we also observed elevated protein levels of MLKL and RIPK3 most pronounced in isolated intestinal epithelial cells of infected Casp $8^{\triangle I E C}$ mice (Figure S7B). In order to provide direct functional evidence for a role of necroptosis in the lethal outcome of Casp $8^{\triangle I E C}$ mice, we subjected Casp $8^{\triangle I E C} M / k l^{-1-}$ and Casp $8^{\Delta / E-}$ ${ }^{C}$ Ripk3 $^{-1-}$ double deficient mice to Salmonella infection. The high lethality and severe body weight loss of Casp $8^{\triangle I E C}$ mice could be completely rescued by additional deletion of MLKL or RIPK3 (Fig. 6b, c, e, f). Histological analysis further revealed minor or no epithelial erosions in both double deficient strains, as demonstrated by $\mathrm{H} \& \mathrm{E}$ and $\beta$-Catenin staining (Fig. $6 \mathrm{~g}, \mathrm{~h}, \mathrm{~S} 8$ ). In line with this, the number of dying IECs, indicated by TUNEL staining, was clearly reduced in animals lacking additionally MLKL or RIPK3 (Figure $\mathrm{S} 8 \mathrm{~A}+\mathrm{C})$. In summary these data demonstrate that epithelial Caspase-8 orchestrates epithelial apoptosis and prevents RIPK3 activation in response to Salmonella Typhimurium infection.

Among the large variety of pro-inflammatory cytokines which are expressed in the mucosa of Salmonella Typhimurium infected mice, several of these are candidates for triggering non-apoptotic cell death in the Caspase- 8 deficient epithelium. ${ }^{2}$ Previously, we have elucidated that LPS induced intestinal epithelial necroptosis is strictly dependent on TNF-a signaling, suggesting a potential contribution of TNF-a to barrier breakdown in Casp $8^{\triangle I E C}$ mice following infection. ${ }^{25}$ Indeed, we observed high expression levels of Tnfa mRNA transcripts following infection with Salmonella Typhimurium in vivo (Fig. 7a). Notably, TNF-a was not only released by lamina propria cells, but also IECs as demonstrated by elevated Tnfa expression in infected organoids (Fig. 7b). To provide direct functional evidence for TNF-a, we infected Casp $8^{\triangle I E C}$ Tnfr $1^{-1-}$ double deficient mice. Additional deletion of Tnfr1 resulted in significantly less severe decrease in body weight and prolonged survival of Casp $8^{\triangle I E C} \operatorname{Tnfr}^{-1-}$ mice (Fig. $7 c, d$ ). However, histological analysis revealed a similar degree of tissue destruction with epithelial erosions in the colon irrespective of the presence or absence of TNF-receptor 1 (Fig. 7e). This was further confirmed by staining for the cell death marker TUNEL and $\beta$-Catenin to visualize barrier integrity (Fig. 7e). These data indicate that Salmonella Typhimurium induced colitis is partially dependent on this pathway and other cytokines might contribute, too.

\section{DISCUSSION}

Programmed cell death plays a crucial role for the regulation of inflammation and infection. Apoptosis and necroptosis are two pathologically relevant types of regulated cell death that strongly influence the pathogenesis of gastrointestinal disorders including inflammatory and infectious diseases. ${ }^{34}$ The cysteine protease Caspase- 8 is a central regulator of both apoptosis and necroptosis. Accordingly, we have previously demonstrated that excessive activation of Caspase- 8 facilitates apoptotic cell death which can culminate in a general destruction of the intestinal barrier, whereas lack of Caspase-8 activity renders IECs susceptible to necroptosis. ${ }^{26,35}$ Here, we provide compelling evidence that the negative regulation of necroptosis by Caspase- 8 plays a fundamental role during enteric infection. We identified that epithelial Caspase-8 is essential to promote host resistance to Salmonella Typhimurium by orchestrating the gut response to control bacterial burden and prevent tissue colonization.

Programmed cell death in the context of host-pathogen interactions highlights the evolutionary conserved capacity of host cells to commit suicide during pathogen infection. This premature death of infected cells perhaps represents the most ancient defense strategy against lethal infection by limiting replication, colonization, and accompanied dissemination by alerting neighboring cells to impending infection. ${ }^{9,14,36}$ Accordingly, in this study we observed elevated levels of cell death mediators in IECs as a response to direct host-pathogen interaction. Importantly, we identified that key players of both the apoptosis and necroptosis pathways were significantly upregulated in the epithelium following Salmonella Typhimurium infection. These data also suggest that RIPK3-mediated necroptosis has been developed as an alternative, non-apoptotic form of epithelial cell death to ensure elimination of infected cells. Indeed, we observed that in case of Caspase- 8 deficiency, IECs undergo RIPK3 and MLKL mediated necroptosis in response to Salmonella Typhimurium infection. While barrier function could be maintained under Caspase- 8 proficient conditions, lack of Caspase- 8 resulted in excessive tissue injury, barrier disruption, and death of mice. Additional deletion of MLKL or RIPK3 was sufficient to block excessive intestinal epithelial necrosis in Casp $8^{\triangle I E C}$ mice. These data suggest that expression of both necroptosis mediators particularly in the intestinal epithelium drives lethality of Casp $8^{\triangle I E C}$ mice. Importantly, while these data clearly indicate that necroptosis may represent a failsafe mode of innate immunity to preserve expulsion of infected cells and thus to control infection, we could not observe differences in the abundance of epithelial cell death in $\mathrm{Mlkl}^{-1-}$ or Ripk3 ${ }^{-1-}$ mice compared to control littermates. Thus in case of elimination of infected epithelial cells, Caspase- 8 mediated apoptosis appears to be the preferred type of epithelial cell death, while necroptosis could represent a safeguard to ensure bacterial clearance. Indeed another study has demonstrated that Ripk $3^{-1-}$ Casp $8^{-/-}$double deficient mice exhibited significantly elevated bacterial burdens and stool scores after oral infection with the mucosal pathogen Citrobacter rodentium. ${ }^{37}$ Similar to our data it has been demonstrated that necroptosis is crucial to limit the pathological inflammation induced by Staphylococcus aureus. Accordingly, necroptosis has been described in macrophages during Staphylococcus aureus induced pneumonia ${ }^{38}$ and in human keratinocytes infected with $S$. aureus. ${ }^{39}$ Moreover, following challenge with Serratia marcescens, S. aureus, Streptococcus pneumoniae, Listeria monocytogenes, and uropathogenic Escherichia coli macrophages, pretreated with inhibitors of RIPK1, RIPK3, or MLKL were protected against death. ${ }^{40}$ Similarly, macrophages from Casp $^{-1-}$ Ripk $^{-1-}$ or Ripk $1^{-1-}$ mice were remarkably resistant to cell death induced by Yersinia pestis and Yersinia pseudotuberculosis, but not Ripk3 deficient macrophages. ${ }^{41}$ Notably, in sharp contrast to our data, macrophages deficient for Caspase-8 alone or in addition with RIPK3 were not resistant to cell death induced by Salmonella Typhimurium. $^{32,41}$ It is currently believed that Salmonella Typhimurium predominantly triggers pyroptosis of infected macrophages. $^{41}$

Interestingly, just recently it has been demonstrated that epithelial NLRC4 inflammasome is sufficient to significantly reduce Salmonella colonization in the cecum and translocation to mesenteric lymph nodes, liver and spleen. Of note it has been reported that Caspase-8 is recruited to the NLRC4 inflammasome upon Salmonella infection of macrophages. However, Caspase-8 deficiency in this setting did not affect cell death, possibly because of compensation of Caspase-1. Our results now suggest that in the intestinal epithelium Caspase-1 is insufficient to compensate for the loss of Caspase-8, since massive intestinal cell death in the 
Caspase-8 deficient epithelium was not associated with increased activation of Caspase- 1 and could be blocked by additional deletion of MLKL and RIPK3 but not pharmacological inhibition of Caspase-1. Notably, Casp $1^{-1-}$ Ripk $^{-1-}$ double deficient mice showed similar disease activity (bacterial burden) in response to Salmonella infection compared to Casp ${ }^{-1-}$ Ripk $^{-1-}$ mice. ${ }^{20}$ Interestingly, mice lacking both caspases in addition to Ripk3 (Casp $1^{-1-}$ Casp ${ }^{-1-}$ Ripk $^{-1-}$ ) showed elevated tissue bacteria loads similar to our study. While these data are derived from full-knock out animals, they still support our main finding that epithelial Caspase-8 is essential to maintain intestinal barrier function by preventing necroptosis and controlling Salmonella colonization.

The release of intracellular contents from necrotic cells into the extracellular milieu not only initiates inflammatory reactions but also influences surrounding cells to activate the cell death machinery. By taking advantage of the organoid model we identified that the early epithelial inflammatory response to bacterial infection includes the expression of many factors, such as TNF- $a$, that are well known for their capability to activate apoptosis and necroptosis. ${ }^{23,25,42}$ These data suggest that cytokines may contribute to barrier breakdown in Casp $8^{\triangle I E C}$ mice. Accordingly, we and others have previously shown that LPS-triggered epithelial apoptosis and necroptosis require the release of TNF-a by mucosal immune cells. ${ }^{26,43,44}$ Here, we uncovered that additional deletion of TNF receptor was sufficient to significantly improve survival but could not rescue lethality of Casp $8^{\triangle I E C}$ mice following Salmonella Typhimurium infection. Similarly, depletion of TNF-a using a neutralizing antibody failed to prevent lethality of mice with an intestinal epithelial cell specific deletion of the Caspase-8 inhibitor cFLIP (cFlip $\left.{ }^{\mathrm{i} \Delta I E C}\right) .^{35}$ Moreover, while Casp $8^{\Delta I E C}$ Tnfr $^{-\prime-}$ mice are protected from LPS-induced septic shock and mortality, inhibition of this signaling pathway is insufficient to block Paneth cell death or inflammation in these mice. ${ }^{25,35}$ This suggests that activation of epithelial apoptosis or necroptosis either occurs by a TNF-a independent pathway or that redundant signals are present during Salmonella Typhimurium infection. Further studies are required to address the contribution of other cytokines on intestinal homeostasis during enteric infection.

Collectively, our data uncover a crucial role for epithelial Caspase-8 in preventing Salmonella Typhimurium induced enteritis. Importantly, while Caspase- 8 has been shown previously to predominantly influence inflammasome regulation in response to Salmonella, we observed that Caspase- 8 is dispensable for this process in IECs. Of note, we provide solid evidence that epithelial Caspase- 8 is an essential factor at the cross road between cell survival and death during Salmonella Typhimurium infection. We therefore propose that Caspase- 8 functions at a convergent point of multiple host defense mechanisms that strongly depend on the cellular context.

\section{MATERIALS AND METHODS}

Mice

Ripk3 ${ }^{-1-45} \mathrm{Mlkl}^{-1-23}$ Casp $^{\triangle \mathrm{IEC}}{ }^{26}$ Casp $^{\triangle \mathrm{IEC}}$ Ripk3 $^{-1-35}$ and Casp8$\triangle \mathrm{IECTnfr}-\mathrm{I}_{-35}$ were described earlier. Casp $8^{\triangle \mathrm{IEC}} \mathrm{Mlkl}^{-l^{\prime}-}$ were generated by crossing $\mathrm{MlkI}^{-1-}$ mice to Casp $8^{\triangle \mathrm{IEC}}$ mice. Throughout the manuscript, we used littermate controls to exclude straindependent differences in susceptibility. Mice were routinely screened for pathogens according to FELASA guidelines. Animal protocols were approved by the Institutional Animal Care and Use Committee of the Regierung von Unterfranken.

Salmonella Typhimurium infection

The S. enterica serovar S. Typhimurium strains UK1 (bioluminescent, provided by Dr. Christian Riedel, University of Ulm), ${ }^{46}$ $\triangle \operatorname{aro}^{28}$ and a GFP expressing wild-type $\operatorname{strain}^{47}$ were cultured at $37^{\circ} \mathrm{C}$ in Luria-Bertani broth, supplemented with erythromycin, streptomycin or ampicillin respectively, under continuous shaking and aeration.

For in vivo infection mice were treated with a single dose of streptomycin by oral gavage $24 \mathrm{~h}$ before infection. Prior to infection, mice were starved for $8 \mathrm{~h}$ and subsequently orally gavaged with $10^{9}$ colony-forming units of Salmonella. For in vivo experiments mice were infected with either the $S$. Typhimurium strains UK1 (Fig. 4a, b and S4) or the attenuated $\triangle a r o A$ strain (Figs. 1, 2, 3, 5, 6, 7, S1, S2, S3, S4, S5, S6, S7 and S8).

For in vitro infections organoids were rinsed from Matrigel, washed, and incubated with a GFP expressing wild-type strain, in crypt culture medium without antibiotics, for $30-45 \mathrm{~min}$ at $37^{\circ} \mathrm{C}$. After washing, the organoids were seeded in Matrigel and cultured in medium containing penicillin, streptomycin, and gentamycin. In some experiments organoids were co-incubated with Belnacasan (VX-765) a potent and selective inhibitor of Caspase-1 (50 $\mu \mathrm{M}$, Selleckchem). Organoid viability was monitored by light microscopy and propidium iodide ( $\mathrm{PI}, \mathrm{BD}$ Bioscience) staining (Life technologies).

\section{Bioluminescent in vivo imaging}

For in vivo imaging, mice infected with the bioluminescent UK1 strain of Salmonella Typhimurium, were anaesthetized with $2 \%$ isoflurane in oxygen and imaged using the IVIS Lumina II. Bioluminescent counts were recorded for $150 \mathrm{~s}$ from the abdomen as region of interest. Images were obtained and analyzed using LIVING IMAGE software (Xenogen).

\section{Immunoblotting}

Proteins were isolated from either whole colon tissue or epithelial scrapings using cell lysis buffer supplemented with PMSF (both Cell Signaling). Proteins were separated using MiniProtean-TGX gels (Bio-Rad) and transferred to a nitrocellulose membrane (BioRad). Membranes were blocked and probed with primary antibodies (for detailed information see Supplementary Table 2) overnight. HRP-linked antirabbit (Cell Signaling, \#7074) was used as a secondary antibody. Blots were developed by chemiluminescence using an ECL substrate (Perkin Elmer or Merck Millipore).

Histology and immunohistochemistry

Histopathological analyses were performed on formalin-fixed paraffin-embedded tissue cross sections (terminal ileum, colon, and cecum) after Mayer's haematoxylin and eosin (H\&E) staining. Immunofluorescence of tissue sections (colon and cecum) was performed using the TSA Cy3/Fluorescein system as recommended by the manufacturer (Perkin Elmer). Primary antibodies (for detailed information see Supplementary Table 2) were incubated overnight. Nuclei were counterstained with Hoechst 3342 (Invitrogen). Cell death (TUNEL) was analyzed using the In Situ Cell Death Detection Kit (Roche). Staining for immune cells was performed on cryosections. For immunohistochemical staining of the mucus layer, samples were fixed in Methanol-Carnoy's fixative. ${ }^{48}$ Images were obtained using the microscope LEICA DMI $4000 \mathrm{~B}$ together with the LEICA DFC360 FX or LEICA DFC420 C camera and the imaging software "LAS AF" (Leica, Wetzlar, Germany).

Gene expression analysis

Total RNA was extracted from intestinal tissue using the peqGOLD Total RNA Kit and from organoids using the peqGOLD Microspin Total RNA Kit (Peqlab, Erlangen, Germany). cDNA was synthesized by reverse transcription using the SCRIPT CDNA Synthesis Kit (Jena Bioscience) and analyzed by real-time qPCR using SYBRGreen reagent (Roche), the LightCycler 480 (Roche) and specific QuantiTect Primer Assays (Qiagen) (for detailed information see Supplementary Table 1). Experimental values were normalized to levels of the housekeeping gene hypoxanthine guanine phosphoribosyl transferase (Hprt). 
Statistical analyses

Differences were compared by Student $t$-test ( 2 groups) using GraphPad Prism 5, GraphPad Software Inc, (San Diego, CA). Quantitative results are presented as mean values with standard deviation (SD). $P<0.05$ was considered significant $\left({ }^{*} P<0.05\right.$; $\left.{ }^{* *} P<0.01 ;{ }^{* *} P<0.001\right)$.

\section{ACKNOWLEDGEMENTS}

The authors thank H. Dorner, M. Zeitler, S. Gößwein, S. Wallmüller and V. Thonn for excellent technical assistance. The authors thank James Murphy (Associated Professor at the Walter and Eliza Hall Institute of Medical Research, Parkville, Victoria, Australia) for providing us with $\mathrm{MIKI}^{-1-}$ mice. This research has received funding from DFG projects within SPP1656 and SFB1181-A08. Further support was given by the Interdisciplinary Center for Clinical Research (IZKF) of the University ErlangenNuremberg.

\section{AUTHOR CONTRIBUTIONS}

C.G. and M.H. designed the research. M.H., I.S., C.G., and B.R. performed the experiments. M.M., SW, G.W.H., M.F.N. provided material that made this study possible. M.H., M.F.N. and C.G. analysed the data and wrote the paper.

\section{ADDITIONAL INFORMATION}

The online version of this article https://doi.org/10.1038/s41385-018-0011-x contains supplementary material, which is available to authorized users.

Competing interest: The authors declare no competing financial interests.

\section{REFERENCES}

1. Kirk, M. D. et al. World Health Organization estimates of the global and regional disease burden of 22 foodborne bacterial, protozoal, and viral diseases, 2010: a data synthesis. PLoS Med. 12, e1001921 (2015).

2. Kaiser, P., Diard, M., Stecher, B. \& Hardt, W. D. The streptomycin mouse model for Salmonella diarrhea: functional analysis of the microbiota, the pathogen's virulence factors, and the host's mucosal immune response. Immunol. Rev. 245, 56-83 (2012).

3. Santos, R. L., Tsolis, R. M., Baumler, A. J. \& Adams, L. G. Pathogenesis of Salmonella-induced enteritis. Braz. J. Med. Biol. Res. 36, 3-12 (2003).

4. Stecher, B. \& Hardt, W. D. Mechanisms controlling pathogen colonization of the gut. Curr. Opin. Microbiol. 14, 82-91 (2011).

5. Carter, P. B. \& Collins, F. M. The route of enteric infection in normal mice. J. Exp. Med. 139, 1189-1203 (1974).

6. Darwin, K. H. \& Miller, V. L. Molecular basis of the interaction of Salmonella with the intestinal mucosa. Clin. Microbiol. Rev. 12, 405-428 (1999).

7. Fink, S. L. \& Cookson, B. T. Pyroptosis and host cell death responses during Salmonella infection. Cell. Microbiol. 9, 2562-2570 (2007).

8. Liss, V. et al. Salmonella entericaremodels the host cell endosomal system for efficient intravacuolar nutrition. Cell Host Microbe 21, 390-402 (2017).

9. Knodler, L. A. et al. Dissemination of invasive Salmonella via bacterial-induced extrusion of mucosal epithelia. Proc. Natl Acad. Sci. USA 107, 17733-17738 (2010).

10. Hybiske, K. \& Stephens, R. S. Exit strategies of intracellular pathogens. Nat. Rev. Microbiol. 6, 99-110 (2008).

11. Friedrich, N., Hagedorn, M., Soldati-Favre, D. \& Soldati, T. Prison break: pathogens' strategies to egress from host cells. Microbiol. Mol. Biol. Rev. 76, 707-720 (2012).

12. Watson, K. G. \& Holden, D. W. Dynamics of growth and dissemination of Salmonella in vivo. Cell. Microbiol. 12, 1389-1397 (2010).

13. Kim, J. M. et al. Apoptosis of human intestinal epithelial cells after bacterial invasion. J. Clin. Investig. 102, 1815-1823 (1998)

14. Sellin, M. E. et al. Epithelium-intrinsic NAIP/NLRC4 inflammasome drives infected enterocyte expulsion to restrict Salmonella replication in the intestinal mucosa. Cell Host Microbe 16, 237-248 (2014).

15. Becker, C., Watson, A. J. \& Neurath, M. F. Complex roles of caspases in the pathogenesis of inflammatory bowel disease. Gastroenterology 144, 283-293 (2013).

16. Jorgensen, I. \& Miao, E. A. Pyroptotic cell death defends against intracellular pathogens. Immunol. Rev. 265, 130-142 (2015)

17. Antonopoulos, $C$. et al. Caspase- 8 as an effector and regulator of NLRP3 inflammasome signaling. J. Biol. Chem. 290, 20167-20184 (2015).

18. Gurung, P. \& Kanneganti, T. D. Novel roles for caspase- 8 in IL-1beta and inflammasome regulation. Am. J. Pathol. 185, 17-25 (2015).
19. Monie, T. P. \& Bryant, C. E. Caspase- 8 functions as a key mediator of inflammation and pro-IL-1beta processing via both canonical and non-canonical pathways. Immunol. Rev. 265, 181-193 (2015).

20. Rauch, I. et al. NAIP-NLRC4 inflammasomes coordinate intestinal epithelial cell expulsion with eicosanoid and IL-18 release via activation of caspase- 1 and -8 . Immunity 46, 649-659 (2017).

21. Robinson, $\mathrm{N}$. et al. Type I interferon induces necroptosis in macrophages during infection with Salmonella enterica serovar Typhimurium. Nat. Immunol. 13, 954-962 (2012)

22. Shutinoski, B. et al. K45A mutation of RIPK1 results in poor necroptosis and cytokine signaling in macrophages, which impacts inflammatory responses in vivo. Cell Death Differ. 23, 1628-1637 (2016).

23. Gunther, $C$. et al. The pseudokinase MLKL mediates programmed hepatocellular necrosis independently of RIPK3 during hepatitis. J. Clin. Investig. 126, 4346-4360 (2016).

24. Jung, H. C. et al. A distinct array of proinflammatory cytokines is expressed in human colon epithelial cells in response to bacterial invasion. J. Clin. Investig. 95 , 55-65 (1995).

25. Gunther, $C$. et al. Caspase- 8 controls the gut response to microbial challenges by Tnf-alpha-dependent and independent pathways. Gut 64, 601-610 (2015).

26. Gunther, C. et al. Caspase- 8 regulates TNF-alpha-induced epithelial necroptosis and terminal ileitis. Nature 477, 335-339 (2011).

27. Sotolongo, J., Ruiz, J. \& Fukata, M. The role of innate immunity in the host defense against intestinal bacterial pathogens. Curr. Infect. Dis. Rep. 14, 15-23 (2012).

28. Grassl, G. A., Valdez, Y., Bergstrom, K. S., Vallance, B. A. \& Finlay, B. B. Chronic enteric salmonella infection in mice leads to severe and persistent intestinal fibrosis. Gastroenterology 134, 768-780 (2008).

29. Hoiseth, S. K. \& Stocker, B. A. Aromatic-dependent Salmonella typhimurium are non-virulent and effective as live vaccines. Nature 291, 238-239 (1981).

30. Zarepour, M. et al. The mucin Muc2 limits pathogen burdens and epithelial barrier dysfunction during Salmonella enterica serovar Typhimurium colitis. Infect. Immun. 81, 3672-3683 (2013).

31. Zhang, K. et al. Age-dependent enterocyte invasion and microcolony formation by Salmonella. PLoS Pathog. 10, e1004385 (2014).

32. Man, S. M. et al. Salmonella infection induces recruitment of Caspase-8 to the inflammasome to modulate IL-1 beta production. J. Immunol. 191, 5239-5246 (2013).

33. Feltham, R., Vince, J. E. \& Lawlor, K. E. Caspase-8: not so silently deadly. Clin. Transl. Immunol. 6, e124 (2017).

34. Gunther, C., Neumann, H., Neurath, M. F. \& Becker, C. Apoptosis, necrosis and necroptosis: cell death regulation in the intestinal epithelium. Gut 62, 1062-1071 (2013).

35. Wittkopf, N. et al. Cellular FLICE-like inhibitory protein secures intestinal epithelial cell survival and immune homeostasis by regulating caspase-8. Gastroenterology 145, 1369-1379 (2013).

36. Jorgensen, I., Rayamajhi, M. \& Miao, E. A. Programmed cell death as a defence against infection. Nat. Rev. Immunol. 17, 151-164 (2017).

37. Gurung, P. et al. FADD and caspase-8 mediate priming and activation of the canonical and noncanonical Nlrp3 inflammasomes. J. Immunol. 192, 1835-1846 (2014).

38. Kitur, K. et al. Toxin-induced necroptosis is a major mechanism of Staphylococcus aureus lung damage. PLoS Pathog. 11, e1004820 (2015).

39. Kitur, K. et al. Necroptosis promotes Staphylococcus aureus clearance by inhibiting excessive inflammatory signaling. Cell Rep. 16, 2219-2230 (2016).

40. Gonzalez-Juarbe, N. et al. Pore-forming toxins induce macrophage necroptosis during acute bacterial pneumonia. PLoS Pathog. 11, e1005337 (2015).

41. Weng, D. et al. Caspase-8 and RIP kinases regulate bacteria-induced innate immune responses and cell death. Proc. Natl Acad. Sci. USA 111, 7391-7396 (2014).

42. Watson, A. J. \& Hughes, K. R. TNF-alpha-induced intestinal epithelial cell shedding: implications for intestinal barrier function. Ann. N. Y. Acad. Sci. 1258, 1-8 (2012).

43. Gunther, C., Buchen, B., Neurath, M. F. \& Becker, C. Regulation and pathophysiological role of epithelial turnover in the gut. Semin. Cell Dev. Biol. 35, 40-50 (2014).

44. Williams, J. M. et al. A mouse model of pathological small intestinal epithelial cell apoptosis and shedding induced by systemic administration of lipopolysaccharide. Dis. Models Mech. 6, 1388-1399 (2013).

45. Newton, K., Sun, X. \& Dixit, V. M. Kinase RIP3 is dispensable for normal NF-kappa $\mathrm{Bs}$, signaling by the B-cell and T-cell receptors, tumor necrosis factor receptor 1 , and Toll-like receptors 2 and 4. Mol. Cell. Biol. 24, 1464-1469 (2004).

46. Riedel, C. U. et al. Construction of p16Slux, a novel vector for improved bioluminescent labeling of gram-negative bacteria. Appl. Environ. Microbiol. 73, 7092-7095 (2007)

47. Valdivia, R. H. \& Falkow, S. Bacterial genetics by flow cytometry: rapid isolation of Salmonella typhimurium acid-inducible promoters by differential fluorescence induction. Mol. Microbiol. 22, 367-378 (1996).

48. Hansson, G. C. \& Johansson, M. E. The inner of the two Muc2 mucin-dependent mucus layers in colon is devoid of bacteria. Gut Microbes 1, 51-54 (2010). 